\title{
Microwave Irradiation: Polar Diels-Alder Reactions using Nitropyrrole and Nitroindole derivates as Electrophiles.
}

\author{
Pedro M.E. Mancini, Anna F. López*, Claudia Della Rosa, María Kneeteman, \\ Guillermo Gamboa
}

Área Química Orgánica- Departamento de Química- Facultad de Ingeniería Química, Universidad Nacional del Litoral. Santiago del Estero 2829. (3000) Santa Fe, Argentina.

FAX: +54-342-4571162. *e-mail:annafrancescalopezbaena@gmail.com

\begin{abstract}
In this work we studied the influence of the microwave irradiation in polar DielsAlder reactions using nitropyrrole and nitroindole derivatives as electrophiles with dienes of different nucleophilicity. The cycloadditions was performed under two conditions with benzene as the reaction medium and solvent free. The results clearly confirm that the use of microwave irradiation in this type of reactions have advantages in relation to the carried out under classical heating. In general the products obtained are similar, but reaction times are lower and the yields increases.
\end{abstract}

Keywords: microwave, nitropyrrole, nitroindole, Diels-Alder, benzene, free-solvent

\section{Introduction}

The microwave-assisted controlled heating has become a powerful tool in organic synthesis, being increasingly used to accelerate organic reactions, increase yields and even reach desired products in minutes and further suppressing the formation of byproducts. ${ }^{1}$ The conventional synthesis is often difficult and time consuming, this way of heating of a reaction mixture is comparatively slow and energy inefficient, since first the heat energy is transferred from the heat source to the reaction vessel, and then the hot surface heats the content of the reaction vessel, due a this the application of microwaves in organic reactions has become popular over the past few years. In many instances microwave heating results in fast reactions, high yields and lower amount of side products. Microwave heating depends on the microwave power absorbing ability of the molecules present in the reaction mixture. Under microwave irradiation the reaction mixture undergoes dielectric heating, which occurring by two form: dipolar 
polarization and ionic conduction. In this way polar and ionic compound can absorb microwave energy very efficiently and are heated up fast. ${ }^{2}$

The Diels-Alder (DA) reaction is one of the most useful tools in organic synthesis. Its potential in heterocyclic chemistry and natural product synthesis is very well known. ${ }^{3}$ Due to our interest in the chemistry of cycloaddition of aromatic heterocyclic compounds substituted with electron withdrawing groups, we have reported studies on the dienophilic character of aromatic systems such as naphthalenes, furans, pyrroles, thiophenes, selenophenes and indoles in their reactions with different dienes developed in benzene as the reaction medium and under conventional heating. These dienophiles reacted with dienes strongly, moderately and poorly activated reactions for obtain in one step the respective cicloadducts. ${ }^{4-8}$ Considering that microwave irradiation (MW) have been used to enhance organic reactions we have realized some experiences with some of these polar process using this methodology. Then, and taken into account the results obtained with the polar Diels-Alder (P-DA) reactions developed in normal thermal conditions, the principal aim of this work is to study the influence of microwave irradiation in the cycloaddition reactions of pyrroles and indoles nitro-substituted, exposed to dienes of different nucleophilicity in the presence of benzene as solvent and complementary in free-solvent conditions.

\section{Results and Discussion}

The study was carried out employing 1-tosyl-2-nitropyrrole 1a, 1-tosyl-3-nitropyrrole 1b, 1tosyl-2-nitroindole $\mathbf{2 a}$ and 1-tosyl-3-nitroindole $\mathbf{2 a}$, as electrophiles and the selected dienes were isoprene $\mathbf{3}$ and 1-trimethylsilyloxy-1,3-butadiene 4. (Figure 1)

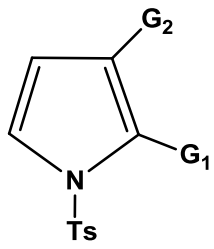

$\begin{aligned} 1 \text { a } \mathrm{G}_{1} & =\mathrm{NO}_{2}, \mathrm{G}_{2}=\mathrm{H} \\ \text { b } \mathrm{G}_{1} & =\mathrm{H}, \mathrm{G}_{2}=\mathrm{NO}_{2}\end{aligned}$

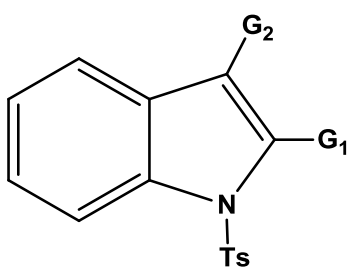

$2 \mathrm{a} \mathrm{G}_{1}=\mathrm{NO}_{2}, \mathrm{G}_{2}=\mathrm{H}$

b $\mathrm{G}_{1}=\mathrm{H}, \mathrm{G}_{2}=\mathrm{NO}_{2}$

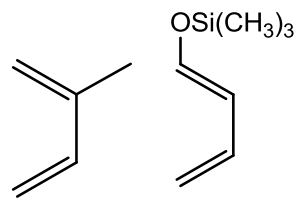

3

Figure 1 
The treatment of the 1-tosyl-2-nitropyrrole $\mathbf{1 a}$ and less reactive isoprene $\mathbf{3}$ reacted under microwave heating following the optimal experimental conditions in the presence of benzene as solvent and in free-solvent conditions, in both cases was obtained the mixture of indoles $\mathbf{5 a}$ and $\mathbf{5 b}$ (Table 1, entries 1 and 2). Similarly the reaction between $\mathbf{1 b}$ and $\mathbf{3}$ showed the mixture of isomeric cycloadducts $\mathbf{5 a}$ and $\mathbf{5 b}$ (Table 1, entries 3 and 4). The reactions carried out in benzene showed lower yield and longer reaction time that the free solvent conditions.

When the $1 \mathbf{a}$ y $\mathbf{1 b}$ reacted with $\mathbf{4}$ under the same conditions, afforded the indole $\mathbf{5 c}$ (Table 1 , entries 5-8), the best results were obtained with free-solvent experience showing high yields. The reaction in the presence of benzene offered moderate yields. However, it is noted in the two experiments that the reaction times were shorter compared to the reactions performed with these electrophiles in normal thermal conditions using benzene as solvent. ${ }^{6-8}$

Table 1. P-DA reactions of $\mathbf{1 a}$ and $\mathbf{1 b}$ with $\mathbf{3}$ and $\mathbf{4}$, under microwave irradiation.

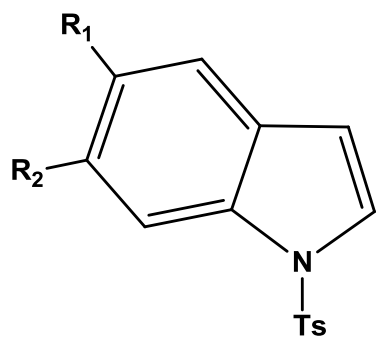

$$
\begin{aligned}
5 \text { a } R_{1} & =\mathrm{CH}_{3}, \mathrm{R}_{2}=\mathrm{H} \\
\text { b } \mathrm{R}_{1} & =\mathrm{H}, \mathrm{R}_{2}=\mathrm{CH}_{3} \\
\text { c } \mathrm{R}_{1} & =H, \mathrm{R}_{2}=\mathrm{H}
\end{aligned}
$$

\begin{tabular}{|c|c|c|c|c|c|}
\hline Entry & Dienophile & Diene & Conditions $^{\mathrm{a}}$ & Results & Yield $^{\mathrm{b}}(\%)$ \\
\hline 1 & 1a & 3,12equiv & Benzene, 120 min & $5 a, 5 b$ & 30 \\
\hline 2 & $1 \mathbf{1 a}$ & 3,12equiv & Free-solvent, $50 \mathrm{~min}$ & $5 a, 5 b$ & 55 \\
\hline 3 & 1b & 3,12equiv & Benzene, $120 \mathrm{~min}$ & $5 a, 5 b$ & 24 \\
\hline 4 & $\mathbf{1 b}$ & 3,12equiv & Free-solvent, $50 \mathrm{~min}$ & $5 a, 5 b$ & 51 \\
\hline 5 & $\mathbf{1 a}$ & 4, 3 equiv & Benzene, $50 \mathrm{~min}$ & $5 c$ & 51 \\
\hline 6 & 1a & 4, 3 equiv & Free-solvent, $20 \mathrm{~min}$ & $5 c$ & 68 \\
\hline 7 & $\mathbf{1 b}$ & 4, 3 equiv & Benzene, $50 \mathrm{~min}$ & $5 c$ & 42 \\
\hline 8 & $\mathbf{1 b}$ & 4, 3 equiv & Free-solvent, $20 \mathrm{~min}$ & $5 c$ & 63 \\
\hline
\end{tabular}

${ }^{a}$ Reaction temperature: $180^{\circ} \mathrm{C}$

${ }^{\mathrm{b}}$ Based on consumed dienophile.

In turn, the reaction of $\mathbf{2 a}$ with the dienes $\mathbf{3}$ and $\mathbf{4}$ using microwave irradiation produce the respective isomeric mixture of carbazoles $\mathbf{6 a}$ and $\mathbf{6 b}$ for the experience with isoprene and the product $\mathbf{6 c}$ was observed when the diene was 1-trimethylsilyloxy-1,3-butadiene. All the products are the result of the nitro group elimination and subsequent aromatization (Table 2, entries 1-4). The reactions of 1-tosyl-3-nitroindole with each diene proceeded similarly to 
dienophile 2a, except that yields were slightly higher for the carbazoles formed (Table 2, entries $5-8)$.

Table 2. P-DA reactions of $\mathbf{2 a}$ and $\mathbf{2 b}$ with $\mathbf{3}$ and $\mathbf{4}$, under microwave irradiation

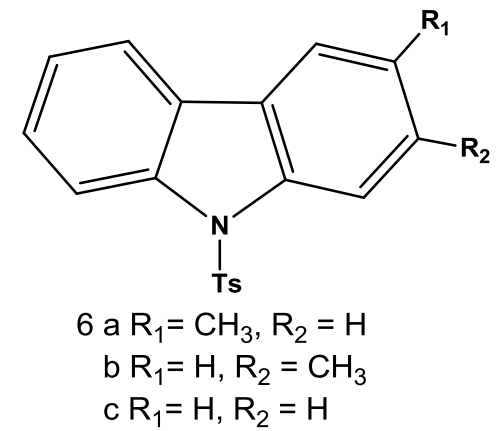

\begin{tabular}{|c|c|c|c|c|c|}
\hline Entry & Dienophile & Diene & Conditions $^{\mathrm{a}}$ & Results & Yield $^{b}(\%)$ \\
\hline 1 & 2a & 3,12equiv & Benzene, $30 \mathrm{~min}$ & 6a, $6 \mathbf{b}$ & 50 \\
\hline 2 & $2 a$ & 3,12equiv & Free-solvent, $30 \mathrm{~min}$ & $6 a, 6 b$ & 62 \\
\hline 3 & $2 \mathbf{b}$ & 3,12equiv & Benzene, 30 min & $6 a, 6 b$ & 57 \\
\hline 4 & $2 \mathbf{b}$ & 3,12equiv & Free-solvent, $30 \mathrm{~min}$ & $6 a, 6 b$ & 67 \\
\hline 5 & 2a & $\mathbf{4 , 3}$ equiv & Benzene, $30 \mathrm{~min}$ & $6 c$ & 61 \\
\hline 6 & $2 \mathbf{a}$ & 4,3 equiv & Free-solvent, $30 \mathrm{~min}$ & 6c & 73 \\
\hline 7 & 2b & $\mathbf{4 , 3}$ equiv & Benzene, $30 \mathrm{~min}$ & $6 c$ & 66 \\
\hline 8 & $2 \mathbf{b}$ & 4,3 equiv & Free-solvent, $30 \mathrm{~min}$ & 6c & 75 \\
\hline
\end{tabular}

${ }^{\mathrm{a}}$ Reaction temperature: $180^{\circ} \mathrm{C}$

${ }^{\mathrm{b}}$ Based on consumed dienophile

\section{Conclusions}

The use of microwave irradiation in this type of P-DA reactions present advantages in relation with those developed in classic conditions although in general the products obtained are similar. In this direction is possible to observe lower reaction times and better yields. In the process developed with nitrated nitropyrroles and nitroindoles it was observed that in all cases the nitro group is responsible to orientation and the selectivity observed and the processes showed nitro extrusion as nitrous acid. It is important to note that microwave irradiation in solvent-free conditions yielded the best results. The importance of this type of experience is related to the reduction of the toxicity and cost, and moreover is a too simple methodology.

\section{Acknowledgments}

This research was supported by CAI+D $2012-\mathrm{N}^{\circ}$ 66,501 20110100478 LI at Universidad Nacional del Litoral, Santa Fe, Argentina 


\section{General Procedure for the MW irradiation reactions}

Microwave irradiation was performed in a Anton Parr Monowave 300, microwave reactor in standard closed vessels. The temperature, the length of the reaction, and the diene/dienophile ratio were dependent on the starting material and are indicated in Table 1 and Table 2.

\section{References and notes}

1. Kremsner, J.M.; Stadler, A. A Chemist`s Guide to Microwave Synthesis. Anton Paar, Austria, 2013.

2. Deb, S.; Wähälä, K. Steroids, 2010, 10.

3. a) Corey, E. J. Angew. Chem., Int. Ed., 2002, 41, 1650-1667. b) Carruthers, W. in Cycloaddition Reactions in Organic Snthesis, Pergamon Press, Oxford, UK, 1990; (b) F. Fringelli, A. Tatichi in The Diels-Alder Reaction, J. Wiley \& Sons, Chichester, UK, 2002.

4. a) Biolatto, B.; Kneeteman, M.; Paredes, E.; Mancini, P.M.E. J. Org. Chem. 2001, 66, 3906-3912. b) Della Rosa, C.; Kneeteman, M.; Mancini, P.M.E. Tetrahedron Lett., 2005, 46, 8711-8714. c) Della Rosa, C.; Kneeteman, M.; Mancini, P.M.E. Tetrahedron Lett., 2007, 48 1435-1438.

5. Mancini, P.; Ormachea, C.; Della Rosa, C.; Kneeteman, M.; Domingo, L. Protic and Nonprotic Ionic Liquids in Polar Diels-Alder Reactions Using Properly Substituted Heterocycles and Carbocycles as Dienophiles. A DFT study" Ionic Liquids. New Aspects for the Future, 2013.

6. a) Della Rosa, C.; Paredes, E.; Kneeteman, M.; Mancini, P.M.E.. Letters in Organic Chemistry, 2004, 1, 369- 371

7. Della Rosa, C.; Kneeteman, M.; Mancini, P. Tetrahedron Lett., 2007, 48, 7075-7078. b) Brasca, R.; Della Rosa, C.; Kneeteman, M.; Mancini, P.M.E. Letters in Organic Chemistry, 2011, 8, 82-87

8. Paredes, E.; Biolatto, B.; Kneeteman, M.; Mancini, P. Tetrahedron Lett., 2000, 41, 80798082. b) Paredes, E.; Biolatto B.; Kneeteman M.; Mancini P.M.E. Tetrahedron Lett., 2002, 43, 4601-4603. c) Paredes E.; Kneeteman M.; Gonzalez Sierra M.; Mancini. Tetrahedron Lett., 2003, 44, 2943-2945. d) Paredes, E.; Brasca, R.; Kneeteman, M.; Mancini, P.M.E. Tetrahedron, 2007, 63, 3790-379. 